\title{
$\$$ Research Square \\ The effect of passive exposure to tobacco smoke on the immune response in children with asthma
}

Agata Wawrzyniak ( $\square$ awawrzyniak@wim.mil.pl )

Military Institut of Medicine https://orcid.org/0000-0003-3255-7623

\section{Agnieszka Lipinska-Opalka}

Military Institut of Medicine

\section{Boleslaw Kalicki}

Military Institut of Medicine

\section{Malgorzata Kloc}

Houston Methodist Research Institute

\section{Research article}

Keywords: environmental tobacco smoke, cytokines, astma, immunology, T-regulatory cells

Posted Date: May 15th, 2020

DOI: https://doi.org/10.21203/rs.3.rs-28878/v1

License: (c) (1) This work is licensed under a Creative Commons Attribution 4.0 International License.

Read Full License

Version of Record: A version of this preprint was published at Substance Use \& Misuse on January 10th, 2021. See the published version at https://doi.org/10.1080/10826084.2020.1869263. 


\section{Abstract}

\section{Introduction}

There are a few publications about the impact of tobacco smoke on the children's immune system.

\section{Material and Methods}

The study group consisted of 43 children with asthma.. The control group consisted of 37 healthy children. The exposure to tobacco smoke was assessed by the presence of the cotinine in the urine.

\section{Results}

The group of children with asthma exposed to tobacco smoke had significantly higher levels of the IL-1 and lower levels IL-4 than children not exposed to the passive smoking. The children from the control group exposed to tobacco smoke had a significantly higher concentration of IL-4 than unexposed children. In the whole analyzed population, there was a significant correlation between the presence of cotinine and the concentration of IL-1 and CRP.

\section{Conclusion}

In this study we found that the passive exposure to tobacco smoke has the immunomodulatory effects on the immune system.

\section{Introduction}

Asthma is a multifactorial disease determined by genetic and environmental factors. An impact of the environment on the course of allergic diseases is extremely important. The environmental component is potentially a modifiable factor, which enables effective primary and secondary prevention.

The GOAL study (Gaining Optimal Asthma ControL) showed that $70 \%$ of patients with asthma, treated with properly selected drugs, can control asthma disease [1]. In addition to the pharmacological intervention, many of the Rother elements influence the control of asthma. According to the current guidelines, the identification of the risk factors of asthma exacerbations is one of the crucial therapeutic goals [2]. When asthma is well-controlled, the presence of even one of these factors increases the risk of future exacerbations. Tens of factors have been described which worsen the course of the asthma disease. Some of these factors such as age, sex, atopy, and ethnicity are unmodifiable, but some can be potentially modified, thus their identification is very important for the therapeutic practice.

An extremely important problem in the pediatric population is passive tobacco smoking, also called the environmental tobacco smoke (ETS). About $80-94 \%$ of ETS represents exposure to the lateral stream of tobacco smoke, and only $6-20 \%$ is the mainstream exposure [3]. 
Exposure to tobacco smoke worsens the lung function and it is particularly dangerous for children with chronic respiratory diseases. There are several mechanisms responsible for the negative impact of ETS on lung function. Many studies suggest that tobacco smoke promotes neutrophilic inflammation in the respiratory tract and the synthesis of the proinflammatory cytokines IL-2 and TNFa [4]. Some studies proved that tobacco smoke may be a direct factor leading to the development of asthma [5]. However, there are only a few publications about the impact of tobacco smoke on the children's immune system.

\section{Aims}

The aim of the study was to assess the impact of passive exposure to tobacco smoke on the immune response in children with asthma.

We evaluated the phenotype of the peripheral blood lymphocytes (CD3, CD4, CD8, CD19, CD16 / 56, CD3 anti HLA-DR), the percentage of natural T-regulatory lymphocytes (CD4 + CD25highCD127-FoxP3 +), and the concentration of the proinflammatory (IL-1, IL-2, IL-6, IL-17A, IL-22, TNFa, IFNY) and anti-inflammatory cytokines (IL-4, IL-10, TGF- $\beta$ ).

\section{Materials And Methods}

The study group consisted of 43 children, 6-18 years old, with asthma, hospitalized in the Department of Paediatrics, Nephrology and Allergology Military Institute of Medicine in Warsaw. The diagnosis of asthma was based on the GINA 2016 criteria. All children had positive skin test results with the common airborne and/or food allergens, which confirmed the atopic source of the disease. During the study, the patients had not presented any symptoms of respiratory tract infection. The gap between testing and exacerbation was no less than 30 days. The control group consisted of 37 healthy children, 6-18 old, without any history of the allergic disease.

All children were subjected to physical examination. The medical interview included information about the exposure to tobacco smoke in the household environment. The concentration of the cotinine in the urine was used as an objective marker to assess the exposure to tobacco smoke.

All parents and patients over 16 years of age gave written informed consent for the participation in the tests.

\section{Exposure to tobacco smoke}

The exposure to tobacco smoke was assessed by the presence of the metabolite of nicotine (cotinine) in the urine. A minimum of $20 \mathrm{ml}$ of the urine sample was collected in a standard $100 \mathrm{ml}$ container from each patient at the first day of hospitalization in the Clinic. The samples were stored at $-20^{\circ} \mathrm{C}$ until further analysis. Cotinine Elisa Kit (Abnova, Taiwan) was used to determine the cotinine concentration according 
to the manufacturer protocol. The limit of detection was $1.0 \mathrm{ng} / \mathrm{ml}$. The cotinine value above $\geq$ $500 \mathrm{ng} / \mathrm{ml}$ was the evidence of active smoking. A value above $\geq 5 \mathrm{ng} / \mathrm{ml}$ suggested passive smoking.

\section{Evaluation of the immunological parameters}

The analysis was carried out as described before [6]. To assess the phenotype of the peripheral blood lymphocytes, the antibodies from the IMK plus test were added to the appropriate cytometric tubes (IMK Test, BD Biosciences, Poland): A - CD45 / CD14, B - isotype control, C - CD3 / CD19, D - CD4 / CD8, E - CD3 / anti-HLA-DR, F- CD16 / 56 / CD3. After adding the erythrocytes lysis solution, the samples were rinsed with buffered saline and subjected to the cytometric analysis.

To characterize natural T-regulatory lymphocytes, cells were stained with antibodies against CD4-PerCP, CD25-APC, CD127-FITC and FoxP3 PE molecules (BD Bioscience, Poland). Cells were collected by flow cytometry. The percentage of nTreg cells (FoxP3 +, CD25high, CD127) was estimed in the CD $4+$ cell population.

To analyze the cytokines, the $50 \mu \mathrm{l}$ beads, $50 \mu \mathrm{l}$ tested serum and $50 \mu$ l detection reagent were added to the cytometric tubes. The samples were incubated for 3 hours at room temperature, in dark.

Subsequently, each sample was rinsed and centrifuged, and the supernatant was collected and washed with $300 \mu$ l of washing buffer. The cytokine level was analyzed using a FACS Calibur flow cytometer.

The measured cytokines were divided into the pro-inflammatory or anti-inflammatory category:

- pro-inflammatory: IL-1, IL-2, IL-6, IL-17A, IL-22, TNFa, IFNy,

- anti-inflammatory: IL-4, IL-10, TGF- $\beta$.

\section{Statistical analysis}

The results were statistically analyzed using the StatSoft software, Inc. software (2014) STATISTICA. The analyzes were initially verified using the normality diagram of the distribution, and the Kolmogorov normality test of Smirnov and Liliefors. The Student's T-test was used to evaluate variables with a normal distribution. For the selected variables inconsistent with the normal distribution, the non-parametric tests, which do not require normality of distribution (U-Mann-Whitney test) were used for the statistical evaluation. A correlation was calculated for variables lacking the normal distribution using the Spearman rank factor. Correlation for the variables with the normal distribution was calculated using Pearson's correlation factor. The $p$-value $<0.05$ was considered as a statistically significant.

\section{Results}

\section{Exposure to tobacco smoke}

In the study group 9 parents declared their nicotinism (21\%) and 34 parents denied smoking in the household. In the group of healthy children, all parents denied smoking. The presence of the cotinine, which indicated exposure to the cigarette smoke was found in 15/42 (36\%) children from the study group 
and in $9 / 37$ (24\%) children from the control group. Statistically significant difference was observed between the results of the interview of nicotinism of people cohabiting with the child and the result of the urine cotinine test.

Median of cotinine concentrations in the group of children with asthma exposed and not exposed to the passive smoking were $22.07 \mathrm{ng} / \mathrm{ml}$ (q25-9.85, q75-28.00) and $0.20 \mathrm{ng} / \mathrm{ml}$ (q25-0.00; q75-0.34). In children from the control group, these values were similar - in people exposed to ETS, the median of cotinine concentration was $26.14 \mathrm{ng} / \mathrm{ml}$ (q25-15.23; q75-31.79), while in children not exposed to ETS $0.12 \mathrm{ng} / \mathrm{ml}$ (q25-0.00, q75-1.19).

\section{The influence of tobacco smoke on the immune system}

The group of children exposed to tobacco smoke had significantly higher levels of the pro-inflammatory IL-1 and lower levels of the anti-inflammatory IL-4 than children not exposed to the passive smoking $(0.41 \mathrm{pg} / \mathrm{ml} \pm 0.58$ vs $0,0.9 \mathrm{pg} / \mathrm{ml} \pm 0.24, \mathrm{p}=0.02$ and $0.68 \mathrm{pg} / \mathrm{ml} \pm 0.77$ vs. $1.19 \mathrm{pg} / \mathrm{ml} \pm 0.72, \mathrm{p}=0.04)$. There was no statistically significant difference in the range of other analyzed immunological parameters (Table 1). 
Table 1

Immunological parameters in children with asthma exposed and not exposed to tobacco smoke.

\begin{tabular}{|llll|}
\hline Immunological parameters & $\begin{array}{l}\text { Study Group ETS(+) } \\
(\text { mean } \pm \text { SD) }\end{array}$ & $\begin{array}{l}\text { Study Group ETS(-) } \\
\text { (mean } \pm \text { SD) }\end{array}$ & p \\
\hline CD3 [\%] & $63.04 \pm 5.77$ & $66.01 \pm 6.67$ & 0.16 \\
\hline CD19 [\%] & $17.38 \pm 5.20$ & $15.78 \pm 4.64$ & 0.31 \\
\hline CD4 [\%] & $32.77 \pm 5.60$ & $34.39 \pm 6.53$ & 0.42 \\
\hline CD8 [\%] & $28.51 \pm 6.07$ & $29.98 \pm 5.19$ & 0.41 \\
\hline CD16/56 [\%] & $14.48 \pm 5.32$ & $12.75 \pm 5.68$ & 0.34 \\
\hline NKT [\%] & $3.34 \pm 2.09$ & $2.99 \pm 1.83$ & 0.58 \\
\hline CD3 anty-HLADR [\%] & $5.88 \pm 2.75$ & $5.45 \pm 2.65$ & 0.63 \\
\hline Treg [\%] & $0.68 \% \pm 0.31$ & $0.69 \% \pm 0.42$ & 0.95 \\
\hline IL-1 [pg/ml] & $0.41 \pm 0.58$ & $0.09 \pm 0.24$ & $0.02 *$ \\
\hline IL-2 [pg/ml] & $2.34 \pm 1.64$ & $1.82 \pm 1.39$ & 0.59 \\
\hline IL-6 [pg/ml] & $2.72 \pm 1.04$ & $2.83 \pm 2.95$ & 0.89 \\
\hline IL-17A [pg/ml] & $7.10 \pm 7.05$ & $7.00 \pm 7.06$ & 0.97 \\
\hline IL-22 [pg/ml] & $12.18 \pm 22.63$ & $14.51 \pm 24.56$ & 0.84 \\
\hline TNFa [pg/ml] & $1.68 \pm 0.45$ & $1.71 \pm 1.58$ & 0.16 \\
\hline IFNy [pg/ml] & $0.71 \pm 1.20$ & $0.54 \pm 0.67$ & 0.76 \\
\hline IL-4 [pg/ml] & $0.68 \pm 0.77$ & $1.19 \pm 0.72$ & $0.04 *$ \\
\hline IL-10 [pg/ml] & $2.65 \pm 2.57$ & $2.21 \pm 1.03$ & 0.48 \\
\hline TGF $\beta$ [pg/ml] & $574.77 \pm 447.49$ & $453.20 \pm 169.95$ & 0.21 \\
\hline
\end{tabular}

The children from the control group exposed to tobacco smoke (ETS+) had a significantly higher concentration of IL-4 than unexposed (ETS-) children ( $4.84 \mathrm{pg} / \mathrm{ml} \pm 8.9 \mathrm{vs} .1 .53 \mathrm{pg} / \mathrm{ml} \pm 0.8, \mathrm{p}=0.05)$. There was no significant difference in the range of other analyzed parameters of the immune response (Table 2). 
Table 2

Elements of the immune response in the group of healthy children exposed and not exposed to tobacco smoke.

\begin{tabular}{|llll|}
\hline Immunological parameters & $\begin{array}{l}\text { Control Group ETS(+) } \\
(\text { mean } \pm \text { SD) }\end{array}$ & $\begin{array}{l}\text { Control Group ETS(-) } \\
(\text { mean } \pm \text { SD) }\end{array}$ & p \\
\hline CD3 [\%] & $64.54 \pm 6.35$ & $59.54 \pm 14.7$ & 0.16 \\
\hline CD19 [\%] & $16.32 \pm 5.13$ & $14.21 \pm 4.8$ & 0.31 \\
\hline CD4 [\%] & $33.65 \pm 6.49$ & $33.79 \pm 6.7$ & 0.42 \\
\hline CD8 [\%] & $27.38 \pm 6.33$ & $30.02 \pm 8.2$ & 0.41 \\
\hline CD16/56 [\%] & $12.82 \pm 4.75$ & $12.45 \pm 6.8$ & 0.34 \\
\hline NKT [\%] & $2.98 \pm 3.07$ & $3.07 \pm 2.9$ & 0.58 \\
\hline CD3 anty-HLADR [\%] & $6.25 \pm 4.06$ & $6.12 \pm 4.0$ & 0.63 \\
\hline Treg [\%] & $0.93 \pm 0.38$ & $1.02 \pm 0.4$ & 0.52 \\
\hline IL-1 [pg/ml] & $0.38 \pm 0.73$ & $0.28 \pm 0.8$ & 0.74 \\
\hline IL-2 [pg/ml] & $1.39 \pm 1.00$ & $3.93 \pm 7.9$ & 0.10 \\
\hline IL-6 [pg/ml] & $4.97 \pm 9.43$ & $2.31 \pm 1.0$ & 0.36 \\
\hline IL-17A [pg/ml] & $7.09 \pm 7.0$ & $5.33 \pm 4.2$ & 0.50 \\
\hline IL-22 [pg/ml] & $12.18 \pm 22.6$ & $8.37 \pm 15.4$ & 0.67 \\
\hline TNFa [pg/ml] & $1.68 \pm 0.4$ & $3.08 \pm 5.3$ & 0.31 \\
\hline IFNy [pg/ml] & $0.71 \pm 1.2$ & $0.79 \pm 0.8$ & 0.86 \\
\hline IL-4 [pg/ml] & $1.53 \pm 0.8$ & $4.84 \pm 8.9$ & $0.05 *$ \\
\hline IL-10 [pg/ml] & $2.65 \pm 2.6$ & $1.85 \pm 0.3$ & 0.36 \\
\hline TGF 3 [pg/ml] & $574.77 \pm 447.49$ & $536.75 \pm 277.3$ & 0.58 \\
\hline
\end{tabular}

In the whole analyzed population, there was a significant correlation between the presence of cotinine and the concentration of IL-1 and CRP (Fig. 1-2).

\section{Discussion}

The interactions between the immune cells play a role in the control of asthma and determine the therapeutic effect of medications. It is believed that the primary function in coordinating the allergic reaction is played by the CD4 + lymphocytes, which are the important source of the Th2 cytokines [7]. The Th2 lymphocytes activate and stimulate the production of the IgE-class antibodies by the B-lymphocytes. The prevalence of the Th2 lymphocytes over the Th1 lymphocytes (responsible for the proinflammatory 
processes) has become the basis for the "hygienic hypothesis". Here, were assessed the immunophenotype of the peripheral blood lymphocytes and the serum cytokine levels. Due to the decisive effects of the interaction between the immune cells on the trajectory of asthma, there has been an increased interest in the agents, which influence the stages of allergic reactions. Here we evaluated the impact of the passive exposure to tobacco smoke (ETS) on the immunological parameters of children with asthma.

In our study, the exposure to tobacco smoke was established by the presence of cotinine in the urine and the interview response of the people cohabiting with a child. We observed that, frequently, the cotinine test indicated the ETS exposure, while the parents declared nonsmoking in the household. The cigarette smoking was declared by $21 \%$ of parents from the study group and none of the parents from the control group. The analysis of cotinine in children's urine indicated ETS exposure in $36 \%$ of children with asthma and $24 \%$ of healthy children. The mean cotinine concentration in the urine of children with asthma exposed to tobacco smoke $(22.07 \mathrm{ng} / \mathrm{ml})$ and healthy children exposed to tobacco smoke $(26.15 \mathrm{ng} / \mathrm{ml})$ were not statistically different. These values corresponded to the 10 cigarettes smoked daily by parents [8]. The lack of a statistically significant difference in the cotinine concentrations indicated that the level of exposure to tobacco smoke was the same in the group of children with asthma and the group of healthy children.

The fact that cotinine was found in children from the non-smoking families demonstrates the prevalence of exposure to tobacco smoke in the living environment. According to the data of the Main Sanitary Inspectorate, the passive exposure to tobacco smoke in public places is a relatively frequent phenomenon, in particular in the children playing areas (4\%), state offices and institutions (3\%), schools (3\%), and cultural facilities (2\%) [9].

The harmfulness of smoking is unambiguous. While the negative effect of smoking on the development of cardiovascular, respiratory and cancer diseases is well known, its effect on the immune system response remains vague. The dualistic effect of the tobacco smoke on the immune cells of the innate and acquired responses had been described; cigarette smoking is associated with both the production and inhibition of pro- and anti-inflammatory cytokines [10]. Tobacco smoke causes more than a hundredfold increase in the exposure to lipopolysaccharide (LPS), which promotes the allergic reaction. Exposure to LPS increases the risk of sensitization to allergens in the air and food, which may lead to the development of atopic diseases and the airway hyperreactivity [11]. Thacher et al. [12] attempted to assess the impact of passive smoking on the development of sensitization to the common food and inhalation allergens and the presence of allergy symptoms; the 3316 newborns from the birth cohort were qualified for the study and observed for 16 years. This study proved that the exposure to ETS in infancy, without previous exposure in fetal life, was associated with a higher risk of food allergy and atopic eczema in children under the age of 4 . The surprising result was the lack of increased risk of allergy in the children of smoking pregnant mothers. One of the possible explanations for this phenomenon, proposed by the authors, is the immunosuppressive effect of pregnancy, preventing the development of sensitization. On the other hand, Hancox et al. [13] suggested that exposure to tobacco smoke of the 
parents of allergic children was associated with a lower risk of developing atopy, which was assessed by the positive skin test in response to the common inhalant allergens. In the studies of Hancox et al. [13] the observation period of the birth cohort was longer and lasted 32 years. The results of these prospective studies indicate the need for a more detailed explanation of the impact of passive smoking on the development and course of allergic sensitization. Our study did not show the effect of ETS on the phenotype of peripheral blood lymphocytes in children with asthma exposed and not exposed to tobacco smoke. However, a statistically significant higher concentration of the pro-inflammatory cytokine IL-1 and a lower concentration of anti-inflammatory IL-4 was observed in children with asthma exposed to tobacco smoke in comparison to children not exposed to ETS. IL-4 is the main cytokine involved in the process of allergic inflammation. It is responsible for promoting Th2 cell responses, thus contributing to the increased production of antigen-specific lgE, and eosinophil and mast cell activation [14]. IL-1 is, in turn, the pro-inflammatory cytokine, which affects both the acute and chronic phases of inflammation as well as the innate and acquired response. It also acts as a factor activating $T$ and $B$ lymphocytes, which leads to the increased production of $\operatorname{lgE}[15]$. Also, IL-1, which promotes a Th2-dependent response, may potentiate IgE-mediated degranulation of the mast cells, which indicates its pathogenic effect in asthma [16]. The experimental studies in mice suggested the association between the IL- 1 and the increased neutrophil influx to the inflammatory focus [17]. Considering the above, the results of our study indicate that passive tobacco smoking in children with asthma is associated with the reduction of the eosinophilic type of inflammation in favor of the neutrophilic type of inflammation.

The relationship between nicotinism and elevated IL-1 concentration was also observed by Fu et al. [18]. The study involved 50 adults with asthma, who were divided into two groups depending on the severity of inflammation assessed by the CRP and IL- 6 levels. Patients with elevated rates of inflammation were characterized by a positive history of nicotinism, reduced lung function parameters, and neutrophilic type of inflammation in the airways. Microarray analysis has identified over 449 genes that differed in the expression between the groups. Among them, the expression of genes involved in the IL-1, TNF-a and nuclear NF-кB pathways, responsible for the development of neutrophilic inflammatory reaction, was altered. The latest review of the data on the mechanisms of development of neutrophil inflammation in asthma indicates that the increase in IL-1 is associated with exposure to tobacco smoke and air pollution [19].

Well-documented data from the literature point to the importance of IL-4 in the development of allergic inflammation [20]. IL-4 induces a change of the Th0 phenotype towards Th2, and changes the production of antibodies from IgG towards IgE, which are the key factors in the allergic cascade. In our study, we observed that the healthy children exposed to tobacco smoke had a higher concentration of IL-4 than the children not exposed to ETS. This may indicate a tendency to develop allergic inflammation in the nonatopic children who are passive smokers. These results are consistent with the reports from other laboratories. Merghani et al. [21] have demonstrated that tobacco smoke exposure is associated with increased levels of IL-4 in the serum. Their study was conducted on a group of 135 healthy children who were randomly selected from the three primary schools. Exposure to tobacco smoke was found in 69 of these children. The higher concentration of IL-4 and TNF-a was observed in the serum of children who 
were passive smokers. Moreover, the concentration of cytokines correlated with the degree of ETS - the higher the exposure to ETS, the higher the concentration of cytokines. Also, the meta-analysis, which assessed the impact of ETS on the development of sensitization, indicated a significant correlation, especially in younger children, between passive smoking and the allergy [22]. The results of our study suggest that exposure to tobacco smoke promotes allergic reactions in children of all ages.

The results of our study also suggest that the passive smoking is associated with the promotion of a neutrophilic type of inflammation in children with asthma. It is believed that the inflammation other than the eosinophilic airway inflammation is associated with the more severe course of asthma disease, increased risk of exacerbations, and it is unmanageable by a classic treatment [23]. O'Brien et al. [24] observed that in the group of children with severe and persistent asthma, the dominant type of inflammation in the airways was the neutrophilic inflammation. Just et al. [25] reported that the neutrophilic asthma is the most serious form of the disease in the pediatric population. In this group of children, corticosteroids do not change the natural trajectory of the disease and may not prevent a gradual decrease in lung function [26]. Despite the lack of response to the treatment with glucocorticosteroids, the neutrophilic asthma responses to the macrolide antibiotics [27]. The positive effect of beta-2-agonists as a potential therapeutic method used in this type of inflammation is also being investigated. Reid et al. [28], showed that the addition of the long-acting salmeterol to the inhalation therapy with corticosteroids positively influenced the stabilization of asthma by reducing neutrophil activity. Considering the above, appropriate treatment should be considered for children with asthma exposed to tobacco smoke and vitamin D deficiency. Due to possibllity of worse prognosis, these children should be under special care and may require more frequent follow-up visits.

\section{Conclusion}

In this study we found that the passive exposure to tobacco smoke has the immunomodulatory effects on the immune system. In children with asthma, the passive exposure to tobacco smoke may be a main factor leading to the neutrophilic inflammation associated with the presence of IL-1. On the other hand, passive exposure to tobacco smoke in the population of healthy children may cause an eosinophilic inflammation associated with the presence of IL-4.

\section{Declarations}

\section{Ethics approval and consent to participate}

The study was approved by the Ethics Committees of the Military Institute of Medicine in Warsaw (No. $123 / 14$ consent). All parents and patients over 16 years of age gave written informed consent for the participation in the tests.

\section{Consent for publication}




\section{Competing Interests \\ The authors declares that they have no competing interests.}

\section{Funding}

This research was supported by Military Institut of Medicine grant 1/8865 (323).

\section{Authors' contributions}

AW, ALO, BK participated in designing the study and acquiring or providing the data. AW carried out the statistical analyses and interpreted the data. AW nad ALO drafted the manuscript. BK, MK supervised the work. All authors also contributed to the interpretation of the data. All authors revised the manuscript critically for important intellectual content and read and approved the final manuscript.

\section{References}

1. Bateman E, Boushey $H$, Bousquet J. Can guideline-defined asthma control be achieved? The Gaining Optimal Asthma Control study. Am J Respir Crit Care Med. 2004;170(8):836-44.

2. GINA REPORT. Global strategy for asthma management and prevention. https://ginasthma.org/wpcontent/uploads/2019/01/2018-GINA.pdf. Published 2018.

3. Lipińska-Ojrzanowska A, Polańska K, Wiszniewska M. Smoking at workplace - legislation and health aspect of exposure to second-hand tobacco smoke. Med Pr. 2015;66(6):827-36.

4. Hagiwara E, Takahashi K, Okubo T, et al. Cigarette smoking depletes cells spontaneously secreting Th1 cytokines in the human airway. Cytokine. 2001;14:121-6.

5. Tomasiewicz J, Rączka A, Łuczak E. Tobacco specific IgE in atopic patients. Alerg Astma Immun. 2002;7(1):55-60.

6. Wawrzyniak A, Lipińska-Opałka A, Zdanowski R. Evaluation of selected immunological parameters and the concentration of vitamin D in children with asthma. Case-control study. Cent Eur J Immunol. 2017;42(1):101-6.

7. Zhu J, Paul W. Peripheral CD4 + T-cell differentation regulated by networks of cytokines and transcription factors. Immunol Rev. 2010;238:247-62.

8. Feleszko W, Zawadzka-Krajewska A, Matysiak K, et al. Parental tobacco smoking is associated with augmented IL-13 secretion in children with allergic asthma. J Allergy Clin Immunol. 2006;1(31):97102.

9. Report from a nationwide survey on subject of attitudes towards smoking 
Trząsalska A, Staszyńska., Krassowska U. Report from a nationwide survey on subject of attitudes towards smoking. https://gis.gov.pl > Postawy-Polaków-do-palenia-tytoniu-Raport-2017. Published October, 2017.

10. Arnson $\mathrm{Y}$, Shoenfeld $\mathrm{Y}$, Amital H. Effects of tobacco smoke on immunity, inflammation and autoimmunity. J Autoimmun. 2010;34(3):258-65.

11. Lannero $E$, Wickman M, Bergstrom A. Exposure to environmental tobacco smoke and sensitisation in children. Thorax. 2008;63:172-6.

12. Thacher J, Gruzieva O, Pershagen G, et al. Parental smoking and development of allergic sensitization from birth to adolescence. Allergy. 2016;71(2):239-48.

13. Hancox R, Welch D, Poulton R, et al. Cigarette smoking and allergic sensitization: a 32-year population-based cohort study. J Allergy Clin Immunol. 2008;121(1):38-42.

14. Steinke J, Borish L. Th2 cytokines and asthma - Interleukin-4: its role in the pathogenesis of asthma, and targeting it for asthma treatment with interleukin-4 receptor antagonists. Respir Res. 2001;2(2):66-70.

15. Nambu A, Nakae S. IL-1 and Allergy. Allergol Int. 2010;59(2):125-35.

16. Hultner $L$, Kolsch $S$, Stassen $M$, et al. In activated mast cells, IL-1 up-regulates the production of several Th-2 related cytokines including IL-9. J Immunol. 2000;164:5556-63.

17. Nakae $\mathrm{S}$, Komiyama $\mathrm{Y}$, Yokoyama $\mathrm{H}$, et al. IL-1 is required for alergen-specific Th2 cell activation and the development of airway hypersensitivity response. Int Immunol. 2003;15:483-90.

18. Fu J, Baines K, Wood L. Systemic inflammation is associated with differential gene expression and airway neutrophilia in asthma. OMICS. 2013;17(4):187-99.

19. Chang H, Lee T, Jun J, et al. Neutrophilic inflammation in asthma: mechanisms and therapeutic considerations. Expert Rev Respir Med. 2017;11(1):29-40.

20. Ngoc P, Gold D, Tzianabos A. Cytokines, allergy and asthma. Curr Opin Allergy Clin Immunol. 2005;5(2):161-6.

21. Merghani T, Saced A, Alawad A. Changes in plasma IL4, TNFa and CRP in response to regular passive smoking at home among healthy school children in Kharyoum, Sudan. Afr Health Sci. 2012;12(1):41-7.

22. Smyth J, Starkey C, Vestbo J. CD4-regulatory cells in COPD patients. Chest. 2007;132:156-63.

23. Rosiek-Biegus M, Fal A. Noneosinophilic mechanisms of asthmatic inflammation. Alerg Astma Immun. 2008;13(4):202-7.

24. O'Brien C, Tsirilakis K, Santiago M. Heterogeneity of lower airway inflammation in children with severe-persistent asthma. Pediatr Pulmonol. 2015;50(12):1200-4.

25. Just J, Bourgoin-Heck M, Amat F. Clinical phenotypes in asthma during childhood. Clin Exp Allergy. 2017;47(7):848-55.

26. He X, Simpson J, Wang F. Inflammatory Phenotypes in Stable and Acute Childhood Asthma. Paediatr Respir Rev. 2011;12(3):165-9. 
27. Svenningsen S, Nair P. Asthma Endotypes and an Overview of Targeted Therapy for Asthma. Front Med. 2017;4:158.

28. Reid $D$, Ward $C$, Wang $N$, et al. Possible anti-infl ammatory effect of salmeterol against interleukin-8 and neutrophil activation in asthma in vivo. Eur Respir J. 2003;21:994-9.

Figures

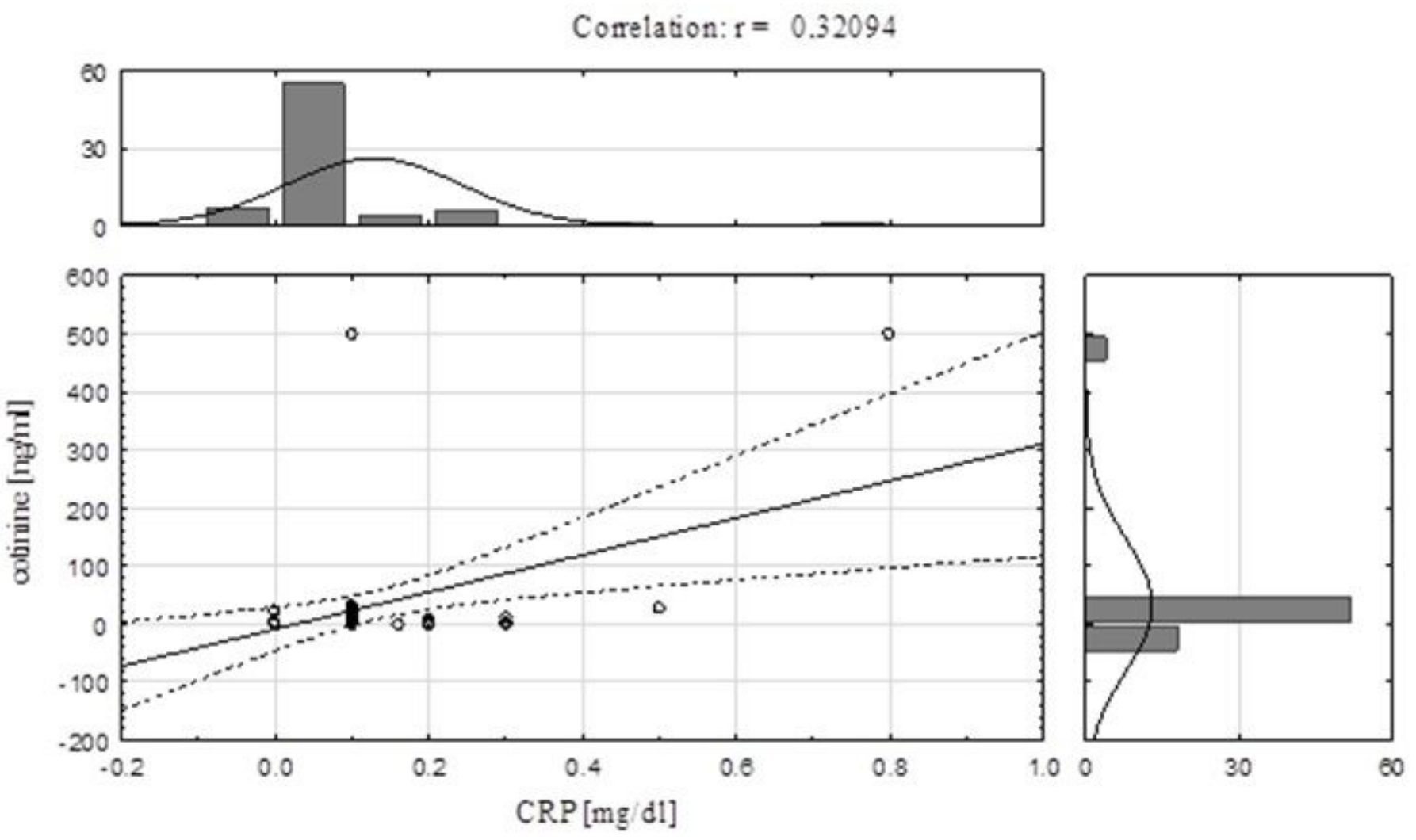

Figure 1

Correlation between cotinine concentration and CRP. 
Correlation: $r=0.05395$
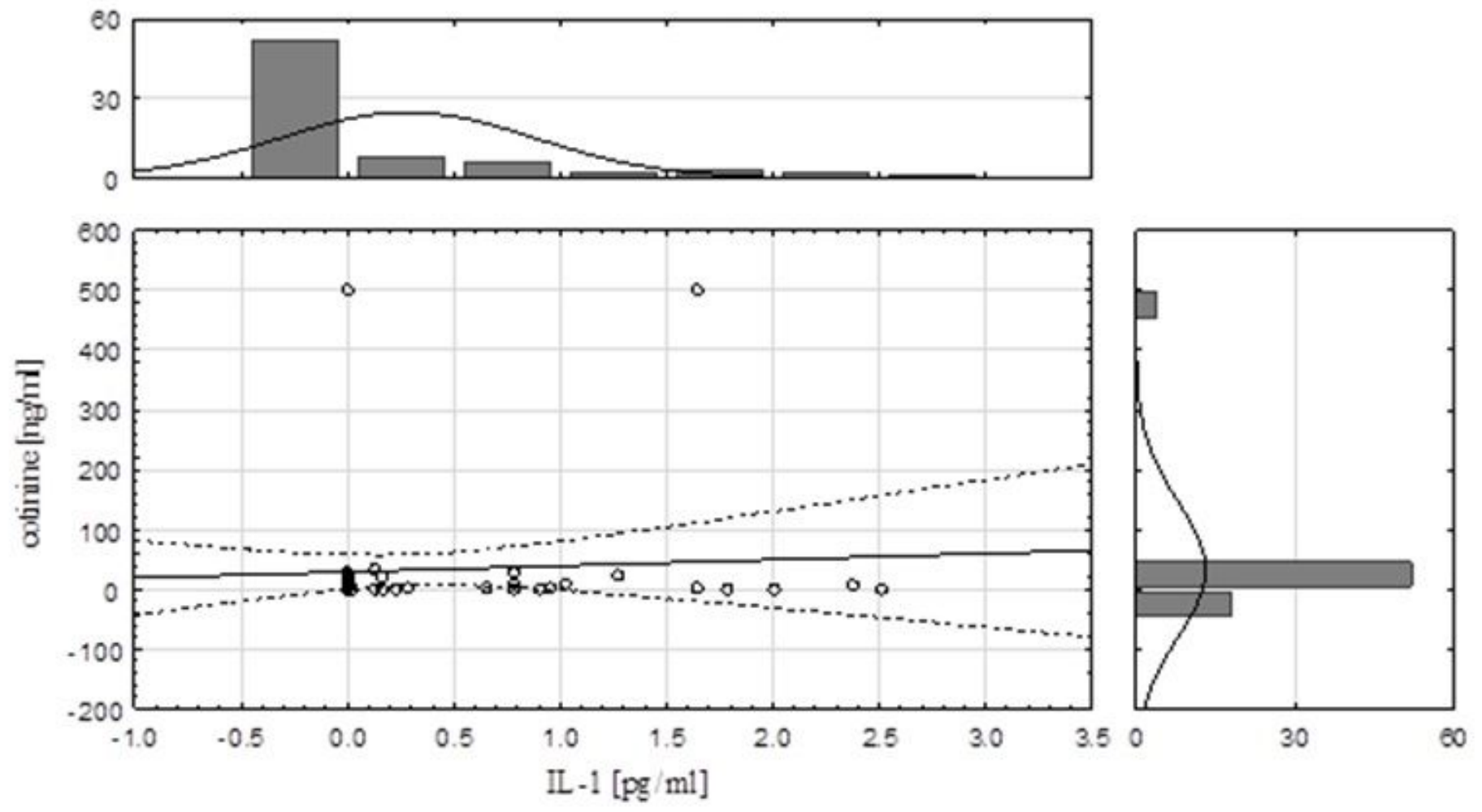

Figure 2

Correlation between cotinine concentration and IL-1. 